\title{
Exact strangeness conservation in heavy ion collisions*
}

\author{
Krzysztof Redlich ${ }^{1, * *}$ and Natasha Sharma ${ }^{2, * * *}$ \\ ${ }^{1}$ Institute of Theoretical Physics, University of Wrocław, 50-204 Wrocław, Poland \\ ${ }^{2}$ Indian Institute of Science Education and Research (IISER) Berhampur, 760010 Odisha, India
}

\begin{abstract}
We investigate the increase in strangeness production with charged particle multiplicity $\left(d N_{c h} / d y\right)$ seen by the ALICE collaboration at CERN in $\mathrm{p}-\mathrm{p}, \mathrm{p}-\mathrm{Pb}$ and $\mathrm{Pb}-\mathrm{Pb}$ collisions using the hadron resonance gas model. The strangeness canonical ensemble is used taking into account the interactions among hadrons using S-matrix corrections based on known phase shift analyses. We show the essential role of constraints due to the exact conservation of strangeness which is instrumental to describing observed features of strange particle yields and their scaling with $d N_{c h} / d y$. Furthermore, the results on comparing the hadron resonance gas model with and without S-matrix corrections, are presented. We observe that the interactions introduced by the phase shift analysis via the S-matrix formalism are essential for a better description of the yields data independent of collision system. This work is based on our longlasting collaboration and most recent publications with Jean Cleymans ${ }^{* * * *}$.
\end{abstract}

\section{Introduction}

The experimental particle yeild data from heavy-ion collisions across different experiments and broad range of energies have been shown to originate from a thermal production [1-7]. These data have been successfully explained by the hadron gas model (HRG) with a common freezeout temperature $T_{f}$ and chemical potentials $\vec{\mu}_{f}$ associated with the conserved charges. It has been shown that the freezeout temperature obtained from the HRG conincides with the chiral crossover temperature from lattice QCD (LQCD) at vanishingly small finite $\mu_{B}$ [8].

It is argued that the S-matrix scheme can improve the HRG model in approximating the QCD partition function in the hadronic phase and hence can provide a more accurate description of the measured particle yields [9-14]. In this work, we apply the S-matrix extended HRG model to analyze data obtained by the ALICE collaboration on charged-particle multiplicity in p-p collisions at $7 \mathrm{TeV}$ [15] as well as $13 \mathrm{TeV}$ [16], in p-Pb collisions at 5.02 $\mathrm{TeV}[7,17]$ and in $\mathrm{Pb}-\mathrm{Pb}$ collisions at $2.76 \mathrm{TeV}$ [18-20], all in the central region of rapidity.

We estimate the yields of (multi-)strange hadrons and discuss strangeness suppression as a function of $d N_{c h} / d \eta$ and strangeness content of hadrons. We formulate the HRG model in the canonical ensemble of strangeness conservation and account for differences between the fireball volume at midrapidity $V_{A}$ and the correlation volume $V_{C}$ required for exact global strangeness conservation [21-24]. We include the S-matrix corrections to proton production

\footnotetext{
*In memory of Jean Cleymans

**e-mail: krzysztof.redlich@uwr.edu.pl

***e-mail: natasha.sharma@cern.ch

****https://home.cern/news/obituary/cern/jean-willy-andre-cleymans-1944-2021
} 
by using the empirical phase shifts of $\pi N$ scattering and the contribution of hyperons to proton yield and corrections to the hyperon yields, employing an existing coupled-channel study involving $\pi \Lambda$, and $\pi \Sigma$ interactions in the $S=-1$ sector [25].

\section{Strangeness production and HRG model}

In the framework of HRG model with the grand canonical ensemble, the quantum numbers are conserved on average and are implemented using the corresponding chemical potentials $\vec{\mu}$ linked to conserved charges $\vec{Q}$. At the LHC energies, all chemical potentials $\vec{\mu}$ are set to zero, i.e. the system is charge neutral. Such a model has been applied to quantify thermalization and particle production in most central heavy-ion collisions at the LHC. At low multiplicity events or low collisions energies, a thermal description requires exact implementation of charge conservation which is usually described in the C-ensemble [21, 22, 26-30].

We focus on the canonical ensemble with exact strangeness conservation (total strangeness $S=0$ ) and assume all other quantum numbers are conserved on average in the GC ensemble with $\vec{\mu}=0$. The strangeness canonical partition function can be expressed as a series of Bessel functions [21, 27, 30, 31],

$$
Z_{S=0}^{C}=\sum_{n, p=-\infty}^{\infty} I_{n}\left(S_{2}\right) I_{p}\left(S_{3}\right) I_{-2 n-3 p}\left(S_{1}\right) .
$$

The mean multiplicity in the canonical ensemble of particle $k$ carrying strangeness $s$ in a given experimental acceptance $A$, are obtained as

$$
\left\langle N_{k}^{s}\right\rangle_{A}=V_{A} n_{k}^{s}(T) \frac{1}{Z_{S=0}^{C}} \times \sum_{n, p=-\infty}^{\infty} I_{n}\left(S_{2}\right) I_{p}\left(S_{3}\right) I_{-2 n-3 p-s}\left(S_{1}\right) .
$$

The particle yields are obtained using the temperature $(T)$ and two volume parameters: the volume of the system in the acceptance $V_{A}$ and the correlation volume $V_{C}$ of global strangeness conservation which appear in the arguments of the Bessel functions. To get the total multiplicity of a given strange particle in the $\mathrm{C}$ ensemble resonance decays have to be added. To identify the contribution and dependence of various terms in Eq. (2) on strange particle quantum number $s$, only leading terms can be considered $(p=n=0)$, so

$$
\left\langle N_{k}^{s}\right\rangle_{A} \simeq V_{A} n_{k}^{s}(T) \frac{I_{s}\left(S_{1}\right)}{I_{0}\left(S_{1}\right)} .
$$

The ratio of $I_{s}\left(S_{1}\right) / I_{0}\left(S_{1}\right)$ is the suppression factor which decreases with increasing $s$ and with decreasing thermal phase-space occupied by strange particles. These correspond to the strangeness canonical suppression that have been introduced [21] to describe thermal production of multi(strange) hadrons in heavy-ion collisions. In this work, we apply the HRG model with the C-ensemble to quantify the production of (multi-)strange hadrons and their behavior with charged particle multiplicity. We modify the HRG model with a more complete implementation of interactions within the S-matrix formalism.

\section{S-matrix formalism and HRG}

The various interactions among hadrons modify the density of states (DOS) of a thermal system and hence the thermal abundances of hadron states [9, 10, 32, 33]. In the scattering matrix (S-matrix) formalism, an effective spectral function $B(M)(M$ represents center-ofmass energy of the system) describing the DOS can be computed from the S-matrix [9]. It summarizes the various interactions among the scattering channels. As the energy increases, 
new interaction channels open and scattering becomes inelastic. The channel-specific spectral function $B_{a}(M)$ describes the energy dependent component of the full $B(M)$ for a given channel $a$. The channel yield, e.g., from a resonance decaying into multiple final states, can be obtained as

$$
n_{a}(T)=\int_{m_{t h}}^{\infty} \frac{d M}{2 \pi} B_{a}(M) n^{(0)}(T, M),
$$

where $n^{(0)}$ is the ideal gas formula for the particle density.

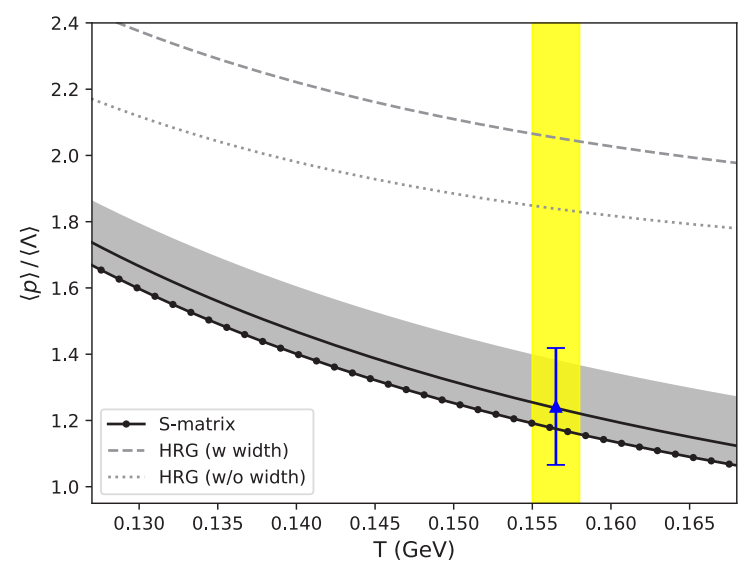

Figure 1. Proton to $\Lambda+\Sigma^{0}$-baryons yield ratio is shown as a function of temperature. The predictions from the S-matrix scheme and from the HRG scheme (with and without width) are presented as line, dashed line and dotted line respectively. The blue solid triangle represents the experimental value measured by the ALICE collaboration for the most central $\mathrm{Pb}-\mathrm{Pb}$ collisions (at $2.76 \mathrm{TeV})$.

We focus on computing the hadron yields of protons and $\Lambda+\Sigma^{0}$-baryons. Following previous studies for protons, we employ the empirical phase shifts from the GWU/SAID PWA on the $\pi N$ scattering [34]. In addition to this, we implement a $\pi \pi N$ background contribution based on the LQCD computation of the baryon charge-susceptibility $\chi_{B Q}$. For the strange $(|S|=1)$ baryon system, we employ an existing coupled-channel model involving $\bar{K} N, \pi \Lambda$, and $\pi \Sigma$ interactions. The S-matrix scheme for $|S|=1$ hyperons is based on Ref. [35] without any extra tuning.

It is observed that the HRG model overestimates the yields of protons and underestimates the yields of $\Lambda+\Sigma^{0}$-baryons. As a result, the ratio of the yields of protons to $\Lambda+\Sigma^{0}$-baryons is larger than the measured experimental ratio. Figure 1 shows the comparison of ratio of protons to $\Lambda+\Sigma^{0}$ yields measured by the ALICE collaboration for the most central $\mathrm{Pb}-\mathrm{Pb}$ collisions (at $2.76 \mathrm{TeV}$ ), with the corresponding predictions from the HRG model and from the $\mathrm{S}$-matrix scheme. It is observed that the result obtained from the measured hadron yields favor the S-matrix prediction and hence illustrates the robustness of this method.

\section{Comparison of results with ALICE data}

We have used the latest version of THERMUS [36] and extended it to include the S-matrix approach. As a first step, we made a fit for each multiplicity bin of pp collisions at $\sqrt{s}=7$ $\mathrm{TeV}$ [15] by keeping the number of parameters to a minimum. Two temperature values were chosen: $T_{f}=156.5 \mathrm{MeV}$ and $T_{f}=160 \mathrm{MeV}$ based on the results from LQCD [37] and the recent HRG model analysis of ALICE data for central $\mathrm{Pb}-\mathrm{Pb}$ collisions [4]. The strangeness suppression factor $\gamma_{s}$ is fixed to $1\left(\gamma_{s}=1\right)$ as motivated by fits in central $\mathrm{Pb}-\mathrm{Pb}$ collisions,. The chemical potentials due to conservation of baryon number and electric charge are set to 
zero for LHC energy. Thus, in the SCE only two parameters remained, the volume of the system in the experimental acceptance $V_{A}$ and the canonical volume $V_{C}$ which quantifies the range of exact strangeness conservation.

Figure 2 (left) shows the hadrons yields calculated using the SCE for different charged particle multiplicities $d N_{c h} / d \eta$ for a single volume $V=V_{A} \simeq V_{C}$. For comparison, the experimental results from the ALICE collaboration are also shown as symbols. The SCE model qualitatively explains the hadron yields data even with a single volume parameter. For large $d N_{c h} / d \eta>100$ all hadron yields, as well as extracted $V$, depend linearly on charged particle rapidity density. However, for lower $d N_{c h} / d \eta$ this dependence is clearly non-linear for strange particles due to strangeness canonical suppression which increases with the strangeness content of particles. At the quantitative level, however, as seen in Fig. 2 (left), using a single volume leads to too much suppression at small charged particle multiplicities, particularly for $S=-2$ and $S=-3$ baryons. This result is consistent with the previous observation, that a single volume canonical model implies too strong strangeness suppression in low multiplicity events [17].

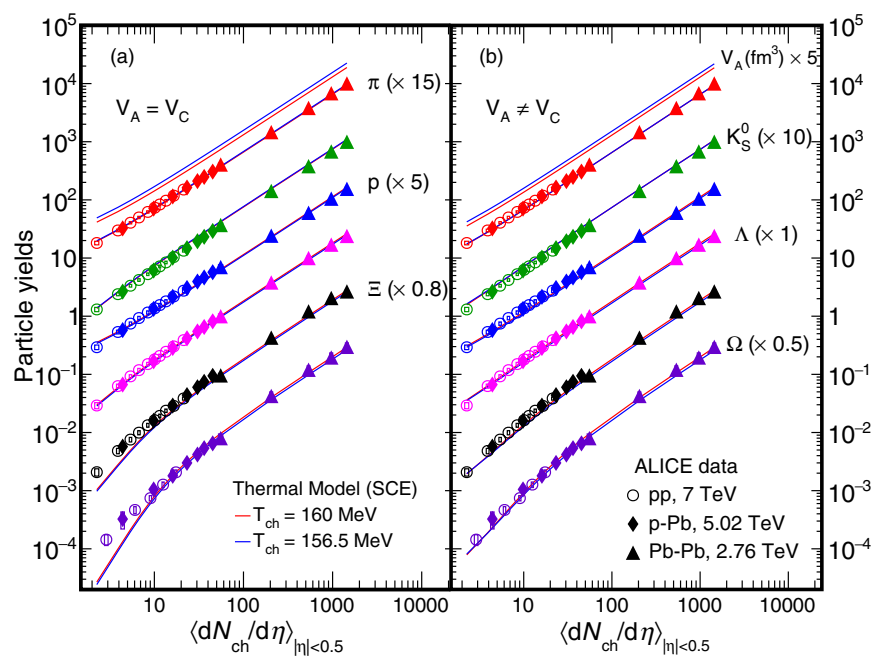

Figure 2. Left: Yields for $V_{A}=V_{C}$. Right: Yields for $V_{A} \neq V_{C}$, The two top lines are the fitted volumes $V_{A}(\mathrm{x} 5)$ in $\mathrm{fm}^{3}$. The particle yields are indicated together with the multiplicative factor used to separate the yields. The solid blue lines have been calculated for $T=156.5 \mathrm{MeV}$ while the solid red lines have been calculated for $T=160 \mathrm{MeV}$. The values of the volumes used have been parametrized empirically (see text for more details).

We have also performed the SCE model fit to data with two independent volume parameters as shown in Fig. 2 (right). In general, strangeness conservation relates to the full phase-space whereas particle yields are measured in some acceptance window. Thus, the strangeness canonical volume parameter $V_{C}$ can be larger than the fireball volume $V_{A}$, restricted to a given acceptance. The resulting yields exhibit much better agreement with data by decreasing strangeness suppression at lower multiplicities due to larger value of $V_{C}$ than $V_{A}$.

The pion yields data are slightly underestimated by the model points while the kaons are overestimated. This has implications for the kaon to pion ratio discussed further below. The 


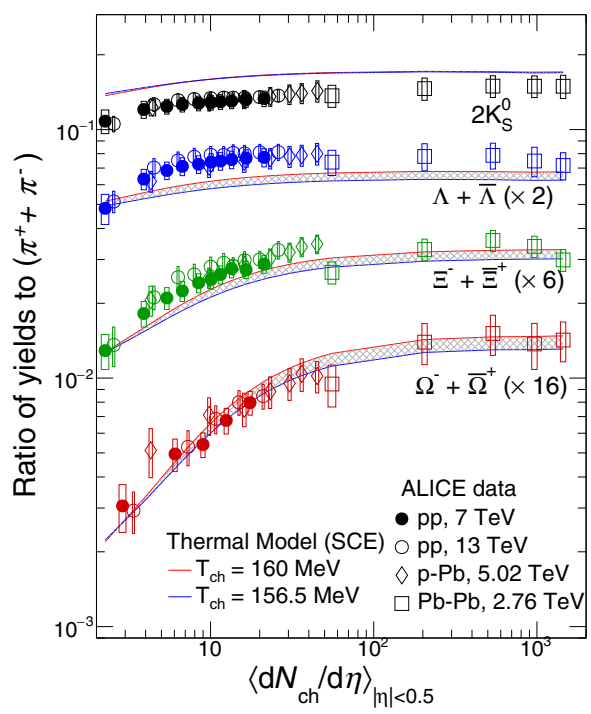

Figure 3. Strange and multi-strange particles to pions yield ratios versus charged particle multiplicity. The symbols show experimental data and the lines represent the SCE model results obtained using $V_{A} \neq V_{C}$.

strangeness suppression effect and its CSE model description are important to describe the dependence of particle yields on the fireball volume $V_{A}$. The ratio of strange particle and pion yields, is shown in Fig. 3. This ratio has been discussed prominently by the ALICE collaboration [15] where a comparison with other model calculations was presented. The SCE model introduced here compares very favorably to the ones discussed in [15]. The underestimation of the pion yield is responsible for the larger discrepancy in the kaon to pion ratio.

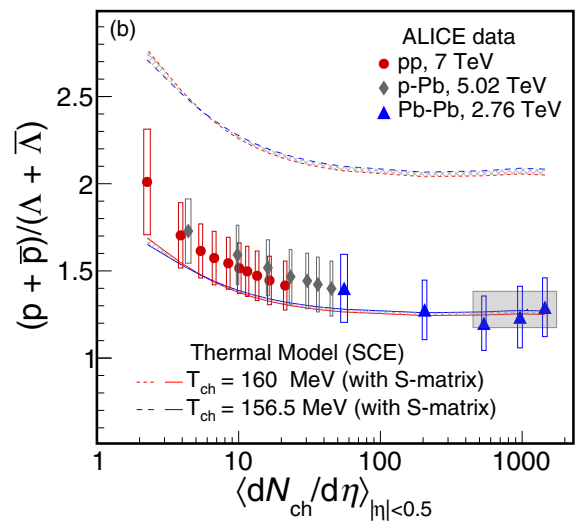

Figure 4. Proton to Lambda ratio as a function of charged particle multiplicity is shown. The lines represent predictions from the HRG model including the S-matrix corrections while the dotted lines represent predictions from the HRG model without $S$-matrix corrections at temperature $\mathrm{T}=160 \mathrm{MeV}$ and $156.5 \mathrm{MeV}$. The solid symbols represent the experimental data measured by the ALICE collaboration[7, 15-20].

The importance of the S-matrix description of proton and hyperon yields can be seen with data from the central $\mathrm{Pb}-\mathrm{Pb}$ collisions by comparing measured $p / \Lambda$ ratio with the $\mathrm{S}$ matrix results as shown in Fig. 4. The lines represent predictions from the HRG model 
including the S-matrix corrections while the dotted lines represent predictions from the HRG model without $\mathrm{S}$-matrix corrections at temperature $\mathrm{T}=160 \mathrm{MeV}$ and $156.5 \mathrm{MeV}$. The shaded region (in gray) shows the theoretical predictions spanned by different stages of the S-matrix improvement: from including only elastic scatterings of ground state hadrons (upper limit) to the full list of interactions (lower limit). The solid symbols represent the experimental data measured by the ALICE collaboration[7, 15-20]. The ratio is very sensitive to the Smatrix corrections which amount to a reduction of proton yield by a factor of 0.75 and a 1.24 enhancement of the $\Lambda$ yield. The thermal model without $S$-matrix corrections exhibits large deviations from the $p / \Lambda$ ratio data. We also found that the ratios of strange and multi-strange particles from SCE model describe the ALICE data very accurately.

\section{Summary}

We have studied the effect of global strangeness quantum number conservation on strangeness production in heavy-ion and elementary collisions in a given acceptance region using the hadron resonance gas (HRG) model in the canonical ensemble. The HRG model is extended with the S-matrix corrections to the yields of protons and hyperons. The S-matrix calculation is based on the empirical phase shifts of $\pi N$ scattering, an estimate of the $\pi \pi N$ background constrained by Lattice QCD results of baryon-charge susceptibility, and an existing coupledchannel model describing the $|S|=1$ strange baryons.

It is demonstrated that an accurate description of the widths of resonances and the nonresonant interactions in a thermal model leads to a reduction of the proton yield relative to the HRG baseline (by $\approx 25 \%$ ). Including the protons from strong decays of $|S|=1$ hyperons, which constitute $\approx 6 \%$ of the total yields, does not alter this conclusion. Such a reduction is also crucial for resolving the proton anomaly in the LHC data. For the hyperons, the Smatrix scheme predicts an increase in the $\Lambda+\Sigma^{0}$ yields relative to the HRG baseline by $\approx 23 \%$. This is consistent with the data from the ALICE collaboration. Furthermore, the S-matrix prediction on the ratio of yields of proton to $\Lambda+\Sigma^{0}$ is in good agreement with the measured values by the ALICE collaboration in $\mathrm{Pb}-\mathrm{Pb}$ collisions in the events with the largest multiplicities $\left(d N_{c h} / d \eta\right)$. The evolution of (multi-)strange baryons to $\Lambda$ yields with $d N_{c h} / d \eta$ calculated in the present thermal model in the $\mathrm{C}$-ensemble follows the measured values within two standard deviations.

A good description was obtained for the variation of the strangeness content in the final state as a function of the number of charged hadrons at mid-rapidity with the same freezeout temperature $T_{f} \sim 156.5 \mathrm{MeV}$. This further supports the idea that at LHC energies (independent of colliding system), the freezeout temperature coincides with the chiral crossover as calculated in LQCD. An exact conservation of strangeness is to be imposed in the full phase-space rather than in the experimental acceptance at mid-rapidity. Consequently, the correlation volume parameter where strangeness is exactly conserved was found to be larger than the fireball volume at mid-rapidity.

The S-matrix formulation discussed here accurately describes the measured hadron yields and supports the interpretation of the LQCD results. It will be useful to employ this in analyzing the multi-strange baryon sectors and the light mesons.

\section{Acknowledgments}

K.R. acknowledges partial support by the Polish National Science Center (NCN) under OPUS Grant No. 2018/31/B/ST2/01663, and by the Polish Ministry of Science. N.S. acknowledges the support of SERB Research Scientist research grant (D.O. No. SB/SRS/2020-21/48/PS) 
of the Department of Science and Technology, Government of India. We also acknowledge fruitful collaboration with Pok Man Lo.

\section{References}

[1] A. Andronic, P. Braun-Munzinger, K. Redlich, J. Stachel, Nature 561, 321 (2018), 1710.09425

[2] A. Andronic, Int. J. Mod. Phys. A 29, 1430047 (2014), 1407.5003

[3] F. Becattini, P. Castorina, A. Milov, H. Satz, Eur. Phys. J. C66, 377 (2010), 0911.3026

[4] A. Andronic, P. Braun-Munzinger, B. Friman, P.M. Lo, K. Redlich, J. Stachel, Phys. Lett. B 792, 304 (2019), 1808.03102

[5] S. Chatterjee, S. Das, L. Kumar, D. Mishra, B. Mohanty, R. Sahoo, N. Sharma, Adv. High Energy Phys. 2015, 349013 (2015)

[6] S. Das, D. Mishra, S. Chatterjee, B. Mohanty, Phys. Rev. C 95, 014912 (2017), 1605.07748

[7] B.B. Abelev et al. (ALICE), Phys. Lett. B 728, 25 (2014), 1307. 6796

[8] A. Bazavov et al., Phys. Rev. D 95, 054504 (2017), 1701.04325

[9] R. Dashen, S.K. Ma, H.J. Bernstein, Phys. Rev. 187, 345 (1969)

[10] R. Venugopalan, M. Prakash, Nucl. Phys. A 546, 718 (1992)

[11] W. Weinhold, B. Friman, W. Norenberg, Phys. Lett. B 433, 236 (1998), nucl-th/9710014

[12] P.M. Lo, B. Friman, K. Redlich, C. Sasaki, Phys. Lett. B 778, 454 (2018), 1710.02711

[13] A. Dash, S. Samanta, B. Mohanty, Phys. Rev. C 99, 044919 (2019), 1806.02117

[14] A. Dash, S. Samanta, B. Mohanty, Phys. Rev. C97, 055208 (2018), 1802.04998

[15] J. Adam et al. (ALICE), Nature Phys. 13, 535 (2017), 1606.07424

[16] S. Acharya et al. (ALICE), Eur. Phys. J. C 80, 167 (2020), 1908.01861

[17] J. Adam et al. (ALICE), Phys. Lett. B 758, 389 (2016), 1512.07227

[18] B. Abelev et al. (ALICE), Phys. Rev. C 88, 044910 (2013), 1303.0737

[19] B.B. Abelev et al. (ALICE), Phys. Rev. Lett. 111, 222301 (2013), 1307.5530

[20] B.B. Abelev et al. (ALICE), Phys. Lett. B 728, 216 (2014), [Erratum: Phys.Lett.B 734, 409-410 (2014)], 1307. 5543

[21] S. Hamieh, K. Redlich, A. Tounsi, Phys. Lett. B 486, 61 (2000), hep-ph/0006024

[22] R. Hagedorn, K. Redlich, Z. Phys. C 27, 541 (1985)

[23] H. Satz, Acta Phys. Polon. Supp. 10, 711 (2017)

[24] P. Castorina, H. Satz, Int. J. Mod. Phys. E 23, 1450019 (2014), 1310.6932

[25] J. Cleymans, P.M. Lo, K. Redlich, N. Sharma, Phys. Rev. C 103, 014904 (2021), 2009.04844

[26] R. Hagedorn (1971)

[27] J. Cleymans, K. Redlich, E. Suhonen, Z. Phys. C51, 137 (1991)

[28] J. Cleymans, H. Oeschler, K. Redlich, Phys. Rev. C 59, 1663 (1999), nucl-th/9809027

[29] C. Ko, V. Koch, Z.W. Lin, K. Redlich, M.A. Stephanov, X.N. Wang, Phys. Rev. Lett. 86, 5438 (2001), nucl-th/0010004

[30] P. Braun-Munzinger, K. Redlich, J. Stachel, Particle production in heavy ion collisions, Quark-Gluon Plasma (World Scientific, 2003), nucl-th/0304013

[31] P. Braun-Munzinger, J. Cleymans, H. Oeschler, K. Redlich, Nucl. Phys. A 697, 902 (2002), hep-ph/0106066 
[32] P.M. Lo, Eur. Phys. J. C 77, 533 (2017), 1707.04490

[33] P.M. Lo, Phys. Rev. D 102, 034038 (2020)

[34] R. Workman, R. Arndt, W. Briscoe, M. Paris, I. Strakovsky, Phys. Rev. C 86, 035202 (2012), 1204.2277

[35] C. Fernandez-Ramirez, I. Danilkin, D. Manley, V. Mathieu, A. Szczepaniak, Phys. Rev. D 93, 034029 (2016), 1510.07065

[36] S. Wheaton, J. Cleymans, M. Hauer, Comput. Phys. Commun. 180, 84 (2009), hep-ph/0407174

[37] A. Bazavov et al. (HotQCD), Phys. Lett. B 795, 15 (2019), 1812.08235 